\title{
Die fernere Lebenserwartung von Rentnern und Pensionären im Vergleich
}

\author{
Ralf K. Himmelreicher, Daniela Sewöster \\ Rembrandt Scholz, Anne Schulz
}

\begin{abstract}
Vergleiche der Lebenserwartung von männlichen Rentnern und Pensionären ab einem Alter von 65 Jahren identifizieren den Einfluss der Lebensbedingungen - hier geht es um Arbeitsbedingungen und Arbeitseinkünfte - auf die Sterblichkeit. Gibt es zwischen verschiedenen Rentner- und Laufbahngruppen Unterschiede bei der ferneren Lebenserwartung? Wie hoch fallen diese aus? Die Unterschiede, die sich aus der Analyse von Mikrodaten des Forschungsdatenzentrums der Rentenversicherung (FDZ-RV) und einer Sonderauswertung des Statistischen Bundesamtes zu den Versorgungsempfängern des öffentlichen Dienstes ergeben, verweisen auf Handlungsbedarf zur Reduzierung sozialer Ungleichheit und ihrer Effekte auf Gesundheit und Lebenserwartung.
\end{abstract}

\section{Einleitung}

Als Folge der seit Mitte des 19. Jahrhunderts steigenden Lebenserwartung erhöht sich der Anteil alter und sehr alter Menschen an der Gesamtbevölkerung. Der demografische Wandel stellt die Gesellschaft vor große Herausforderungen. Ihn zu verstehen, erfordert auch Untersuchungen zur sozialen Ungleichheit der Lebenserwartung in Deutschland. Bisher liegen nur wenige Untersuchungen zur differenziellen Sterblichkeit vor, die auf großen Fallzahlen oder Totalerhebungen beruhen (vgl. Hoffmann et al. 2006). Seit Öffnung der Mikrodaten der gesetzlichen Rentenversicherung (GRV) in Deutschland für die wissenschaftliche Forschung durch das Forschungsdatenzentrum der Rentenversicherung (FDZ-RV) können Studien, die sozioökonomische Differenzen und deren Einfluss auf die Lebenserwartung bei Rentnern untersuchen, durchgeführt werden (vgl. Gaudecker/Scholz 2006; Scholz 2006a; Scholz et al. 2006; Shkolnikov et al. 2007). Diese Studien belegen, dass GRV-Rentner mit höheren Anwartschaften und/oder höherem sozialen Status tendenziell höhere Lebenserwartungen aufweisen.

Diese Befunde stimmen für Deutschland mit auf Survey-Daten beruhenden Ergebnissen überein (Reil-Held 2000) und bestätigen sich überwiegend in internationalen Studien. Für mehrere europäische Länder konnte bereits gezeigt werden, dass die Lebenserwartung mit höherer Bildung und einer höheren beruflichen Stellung steigt (Doblhammer et al. 2005; Mackenbach et al. 2003). Wolfson (1993) wies an- hand von kanadischen Rentendaten einen negativen Zusammenhang zwischen Einkommen und Sterblichkeit nach. ${ }^{1}$ Für Deutschland wurde in mehreren Studien unter Verwendung von Survey-Daten der Zusammenhang zwischen sozioökonomischen Determinanten und Mortalität untersucht: Sie weisen einen positiven Einfluss von hohem Einkommen und guter Gesundheit auf die Lebenserwartung nach (Helmert 2003; Lampert et al. 2007; ReilHeld 2000).

Lassen sich die für die Population der Rentner festgestellten Ergebnisse auch auf andere Gruppen übertragen? Eine statistisch vergleichbare Gruppe ist in diesem Zusammenhang die Gruppe der Bezieher von Ruhegehalt nach beamtenrechtlichen Vorschriften (Pensionäre). Rentner sind in ihrer Erwerbsphase überwiegend sozialversicherungspflichtig beschäftigt gewesen; Pensionäre sind ehemalige Beamte, Berufssoldaten, Richter und Dienstordnungsangestellte im Ruhestand. Luy (2006) zeigte mit den Daten des Lebenserwartungssurveys, dass Beamte im Vergleich zu Arbeitern, Selbstständigen und Angestellten die höchste fernere Lebenserwartung aufweisen. Geringe Fallzahlen in den SurveyDaten verringern allerdings die Robustheit dieser Befunde, insbesondere bei weiterer Differenzierung der Vergleichsgruppen u. a. nach Laufbahngruppen.

Dieser Beitrag basiert auf Daten der deutschen Rentenversicherung und der Sonderauswertung der Statistiken „Personal des öffentlichen Dienstes“ und „Versorgungsempfänger des öffentlichen Dienstes“. Von ihnen werden nahezu alle Männer in Deutschland repräsentiert. Auf Basis der beiden Gesamterhebungen werden erstmalig Mortalitätsunterschiede von männlichen
GRV-Rentenbeziehern und Pensionären in vergleichender Perspektive untersucht.

Im Hinblick auf die Mortalität gehen wir generell davon aus, dass höhere Renten bzw. Laufbahnen die Lebenserwartung ver-

\footnotetext{
Zur internationalen Studienlage, in denen Bezieher höherer Einkommen höhere Lebenserwartungen aufweisen vgl. auch Unger (2003) und Lauterbach (2007).
}

Ralf K. Himmelreicher, Dr., ist Referent am Forschungsdatenzentrum der Rentenversicherung (FDZ-RV) Berlin. Arbeitsschwerpunkte: Alterssicherung im Drei-SchichtenModell, Verteilungsfragen, empirische Wirtschafts- und Sozialforschung. e-mail: ralf.himmelreicher@drv-bund.de Daniela Sewöster, Dipl.-Psych., ist Mitarbeiterin am Forschungsdatenzentrum der Rentenversicherung (FDZ-RV) Berlin. Arbeitsschwerpunkte: empirische Sozialforschung, Bedeutung psychischer Erkrankungen für Erwerbsminderung und Rehabilitation. e-mail: daniela.sewoester@drv-bund.de Rembrandt Scholz, Dr., ist wissenschaftlicher Mitarbeiter und Forschungskoordinator am Rostocker Zentrum zur Erforschung des Demografischen Wandels. Arbeitsschwerpunkte: Mortalitätsentwicklung in Deutschland, Pflegebedarf in der regionalen Differenzierung, Regionale Bevölkerungsprognosen 2020.

e-mail: scholz@rostockerzentrum.de Anne Schulz, BA, ist studentische Hilfskraft am Rostocker Zentrum zur Erforschung des Demografischen Wandels im Forschungsbereich Regionale und Lokale Bevölkerungsprognosen und Planung. e-mail: schulz@rostockerzentrum.de 
längern - bezüglich der sozialen Ungleichheit der Lebenserwartung innerhalb und zwischen Rentnern und Pensionären führen wir erweiternd folgende Thesen ein:

(1) Zwischen den GRV-Rentnern bestehen nach Rentenhöhe nur geringe Sterblichkeitsunterschiede. Sie bilden auf Lebensumstände bezogen im Vergleich zu Pensionären heterogene Renteneinkommensgruppen. Beispielsweise können Bezieher höherer Renten während ihres Erwerbslebens sowohl leitende Angestellte als auch (Akkord-)Arbeiter in der Stahlindustrie gewesen sein. Beide Beschäftigtengruppen dürften aufgrund ihrer Arbeitsbedingungen und Lebensgewohnheiten unterschiedliche Lebenserwartungen aufweisen. Da beide Gruppen ähnlich hohe Renten bei unterschiedlicher Lebenserwartung beziehen können, sinkt die durchschnittliche Lebenserwartung der Gruppe mit höheren Renteneinkommen. Folglich sind die Sterblichkeitsunterschiede nach Renteneinkommeninnerhalb der GRV-Rentner geringer als bei den Beamten.

(2) Zwischen den Pensionären bestehen große Sterblichkeitsunterschiede. Beamtenlaufbahnen orientieren sich streng an schulischer und beruflicher Qualifikation und zwischen den Laufbahngruppen gibt es wenig Durchlässigkeit. Da die Laufbahngruppen, wie der Name suggeriert, die weitere berufliche Entwicklung der früheren Beamten in Bahnen lenkte, gibt es keine mit den GRV-Rentnern vergleichbaren Möglichkeiten der Mobilität zwischen den Laufbahnen bzw. Lebensstilen (z. B. keine Stahlarbeiter im höheren Dienst).

(3) Beamte haben eine höhere Lebenserwartung als GRV-Rentner. Ein Gesundheitstest ist vor der Verbeamtung zwingend erforderlich, insofern findet an dieser Stelle eine Selektion der gesünderen Personen statt. Ferner wird Beamten ein ausgeprägtes Risikovermeidungsverhalten unterstellt, gerade weil ihre hohe Arbeitsplatzsicherheit mit sicheren Einkommen die Kosten riskanten Verhaltens erhöht. Als ökonomisch rationale Akteure versuchen sie, hohe Kosten zu vermeiden und verhalten sich deshalb risikoavers. Generell begünstigt eine planbare Zukunft in Form einer Laufbahn ein gesundheitsbewusstes Verhalten. Dies und das praktizierte betriebliche Gesundheitsmanagement gehen mit höheren Lebenserwartungen einher als bei GRV-Rentnern.
Die Analyse der ferneren Lebenserwartung von Rentnern und Pensionären erfolgt vor dem Hintergrund der drei Thesen in mehreren Schritten. Zunächst werden die beiden Datenbasen und der methodische $\mathrm{Zu}$ gang beschrieben (Abschnitt 2). Im nächsten Schritt geben wir einen Überblick über zentrale Fallzahlen der beiden untersuchten Populationen und vergleichen die ferneren Lebenserwartungen von männlichen Rentnern und Pensionären in Abhängigkeit von ihren Entgeltpunkten bzw. Laufbahngruppen im Zeitverlauf miteinander (Abschnitt 3). Schließlich werden in einem Fazit die wichtigsten Befunde zusammengefasst und sozialpolitische Schlussfolgerungen gezogen (Abschnitt 4).

\section{Datenbasis und Methode}

Die wenigen Untersuchungen zum Thema soziale Ungleichheit der Lebenserwartung in Deutschland basieren meist auf kleinen Stichproben, Teilpopulationen oder auf Befragungsdaten. Aufgrund von hohen jährlichen Schwankungen des Einkommens in Umfragedaten sind diese für Untersuchungen zum Lebensarbeitseinkommen nicht geeignet (vgl. Haider/Solon 2006). Deshalb werden in der vorliegenden Studie Analysen auf Basis von prozessproduzierten Daten der Rentenversicherung und des Statistischen Bundesamtes durchgeführt.

\subsection{DATEN ÜBER RENTNER}

Für $91 \%$ der über 65-jährigen Männer in den alten Bundesländern und $99 \%$ vergleichbarer Männer in den neuen Bundesländern gilt, dass sie eine gesetzliche Rente beziehen (Bieber/Klebula 2005). Die Höhe der Rente ist ein Indikator für das Lebensarbeitseinkommen der Versicherten: Je mehr ein Versicherter im Laufe seines Lebens verdient hat, desto höher sind seine Altersbezüge. Seit 2005 stehen über das FDZ-RV die Mikrodaten des statistischen Berichtssystems der GRV in anonymisierter Form für wissenschaftliche Forschungsprojekte zur Verfügung. ${ }^{2}$ Die Datensätze umfassen insbesondere Informationen zum Rentenbestand und Rentenwegfall und sind damit für Mortalitätsschätzungen besonders gut geeignet. Sie enthalten soziodemografische und sozioökonomi- sche Merkmale der Versicherten und beinhalten mit der Variable „persönliche Entgeltpunkte“ eine Messgröße für das Lebensarbeitseinkommen von rentenversicherten Personen. Insofern können Sterberisiken in Abhängigkeit von der Höhe der eigenen Rente berechnet werden. ${ }^{3}$

Die prozessproduzierten Daten des FDZ-RV sind von sehr hoher Qualität. Im Unterschied zu Befragungsdaten treten bei prozessproduzierten Daten keine Erinnerungsfehler, keine Antwortverweigerungen und keine Probleme durch Panelmortalität auf (Wübbecke 2006, S. 160). Ferner machen die prozessproduzierten Daten Analysen mit sehr hohen Fallzahlen möglich, die die statistische Aussagekraft der Ergebnisse verbessern. Wie Salzmann und Kohls (2007) aufzeigen, sind auf Stichproben basierende Mortalitätsanalysen problematisch. Daher wurde für die folgenden Analysen mit dem Gesamtbestand der Daten gearbeitet. ${ }^{4}$

Die individuelle Rentenhöhe hängt vor allem von dem Beitrag ab, der in den einzelnen Jahren der Erwerbstätigkeit eingezahlt wurde. Der Rentenentgeltpunkt eines Jahres berechnet sich als relatives Verhältnis des persönlichen verbeitragten Jahreseinkommens zum Durchschnitt der Jahreseinkommen aller Beitragszahler. Für ein Einkommen, das dem Durchschnitt entspricht, ergibt sich ein Wert von 1; der Standardrentner erzielt also nach 45-jähriger Beschäftigungszeit 45 Rentenentgeltpunkte. Die Multiplikation der Anzahl der persönlichen Entgeltpunkte mit dem aktuellen Rentenwert ergibt die Höhe der Rente in Euro. Im Unterschied zur Rentenhöhe stellen die persönlichen Entgeltpunkte ein Maß dar, welches standardisiert ist und Zeit- wie Regionalvergleiche ermöglicht. In die Berechnung der persönlichen Entgeltpunkte gehen allerdings neben Zeiten der sozialversicherungspflichtigen Beschäftigung weitere rentenrechtlich bedeutsame Zeiten ein, beispielsweise Lohnersatzleistungen für Arbeitslosigkeit und Krankheit. Vor dem Hintergrund, dass Männer nahe-

\footnotetext{
2 Zum Datenangebot siehe www.fdz-rv.de.

3 Damit werden abgeleitete Renten, wie Witwerrenten, von der Analyse ausgeschlossen. Zu Analysen des Mehrfachrentenbezugs auf Basis von GRV-Daten siehe Hagen et al. (2007).

4 Hierzu konnten wir einen Gastwissenschaftler-Arbeitsplatz und das kontrollierte Fernrechnen am FDZ-RV nutzen. Weitere Ausführungen zu den Nutzungsmöglichkeiten der FDZ-RV-Daten finden sich in Himmelreicher et al. (2006).
} 
zu keine für sie rentenrechtlich relevanten Kindererziehungszeiten ${ }^{5}$ haben und langjährige Erwerbsbiografien bei Männern der untersuchten Kohorten die Regel waren, ist das Merkmal „persönliche Entgeltpunkte" ein valider Indikator für das Lebensarbeitseinkommen. Da es die gesamten Arbeitseinkommen zusammenfasst, kann das Merkmal als die theoretisch relevante Größe für den Zusammenhang zwischen Einkommen und Mortalität bezeichnet werden (Himmelreicher/Gaudecker 2008).

Die Untersuchungspopulation wurde eingegrenzt auf in Deutschland lebende Männer im Alter von 65 Jahren und älter, die eine eigene Rente beziehen. Die Altersabgrenzung wurde deshalb so gewählt, weil im Alter von 65 Jahren das Rentenzugangsgeschehen überwiegend abgeschlossen ist (Himmelreicher 2006, S. 55) und sich das Gros der versicherten Männer dieser Altersgruppe im Rentenbestand, mathematisch gesprochen dem Nenner der Sterblichkeitsberechnungen, befindet. Nach dieser Eingrenzung sind etwa 5,2 Mio. Personen im Rentenbestand 2002 enthalten, denen 256.000 Sterbefälle im Jahr 2003 gegenüberstehen (Tabelle 1).

Die Analyse wurde auf Männer begrenzt, weil die Erwerbsneigung von Frauen insbesondere in den alten Bundesländern in den betrachteten Kohorten vergleichsweise gering ist. Die Annahme, dass die Zahl der persönlichen Entgeltpunkte ein hinreichendes Maß zur Beschreibung ihrer sozialen Situation darstellt, ist damit für Frauen nicht plausibel. Die Zahl der Entgeltpunkte von Männern stellt im Unterschied zu den Frauen wegen ihrer überwiegend langjährigen Erwerbsbiografien eine hinreichende Approximation dar (vgl. Himmelreicher/Frommert 2006).

\subsection{DATEN ÜBER PENSIONÄRE}

Als Äquivalent zum Lebensarbeitseinkommen von Rentnern eignen sich die Laufbahngruppen von Beamten und vergleichbaren Pensionsberechtigten. Die vier Laufbahngruppen (einfacher, mittlerer, gehobener und höherer Dienst) korrespondieren stark mit der höchsten schulischen und beruflichen Qualifikation und bestimmen die Höhe der Besoldung während der Dienstzeit, wobei Beamte des höheren Dienstes die höchste Besoldung beziehen. Grundlage für die Berechnung des Ruhegehalts sind die ruhegehaltsfähigen Dienstzeiten und die ruhegehaltsfähigen Dienst- bezüge, aus denen sich der Ruhegehaltssatz ergibt. Ruhegehaltsfähige Dienstzeiten sind alle Zeiten als Beamter und Zeiten der Tätigkeit im öffentlichen Dienst, soweit sie der Ernennung zum Beamten unmittelbar vorangehen und zu ihr geführt haben. Grundsätzlich anders als im System der gesetzlichen Rentenversicherung, bei dem der gesamte Verlauf der Erwerbsbiografie für die Höhe der Anwartschaft maßgeblich ist (Bäcker et al. 2000, S. 294ff.), errechnen sich die ruhegehaltsfähigen Dienstbezüge aus dem letzten Grundgehalt (zuzüglich berücksichtigungsfähiger Zulagen) unmittelbar vor der Pensionierung. Das Ruhegehalt entspricht, in Abhängigkeit von den ruhegehaltsfähigen Dienstzeiten, einem Anteil von 35 bis $75 \%$ der zuletzt erzielten Besoldung.

Die Laufbahngruppe unmittelbar vor der Pensionierung kann als Hilfskonstruktion für das Lebensarbeitseinkommen eines Beamten herangezogen werden, weil das Beamtenrecht die Erwerbsbiografien und -einkommen von Beamten bestimmt. Im Falle von aufwärtsmobilen Laufbahnwechslern kann davon ausgegangen werden, dass sie der angestrebten Laufbahn entsprechende Lebensgewohnheiten bereits zuvor praktiziert haben.

Unsere Untersuchung zu den Versorgungsempfängern des öffentlichen Dienstes basiert auf einer Sonderauswertung 6 des Statistischen Bundesamtes. Es handelt sich, wie bei den Daten der Rentenversicherung, um valide Daten einer prozessproduzierten Vollerhebung. Die Daten wurden ebenfalls auf Männer beschränkt, die eine eigene Versorgung beziehen, um die Vergleichbarkeit der beiden Populationen zu gewährleisten. Erfasst sind alle Versorgungsempfänger nach Beamten- und Soldatenversorgungsrecht (Beamte, Richter und Soldaten), alle Versorgungsempfänger nach dem Gesetz zu Artikel 131 GG (nach dem zweiten Weltkrieg nicht übernommene Beamte, Berufssoldaten und sonstige Bedienstete mit Beamtenversorgung) und alle Empfänger einer Versorgung nach beamtenrechtlichen Grundsätzen (Bezieher von Amtsgehalt und Angestellte/Arbeiter, die aufgrund einer Dienstordnung beschäftigt waren).

\subsection{OPERATIONALISIERUNG, VERGLEICHBARKEIT UND KAUSALITÄT}

Das Lebensarbeitseinkommen der GRVRentner wird in der vorliegenden Studie mithilfe persönlicher Entgeltpunkte (in Quintilen) operationalisiert und das der Pensionäre im Rahmen der Laufbahngruppen. Beide Messverfahren stellen eine vergleichbare Approximation der Lebensarbeitseinkommen dar. Zu beachten ist dennoch, dass die persönlichen Entgeltpunkte die gesamte sozialversicherungspflichtige Erwerbsbiografie widerspiegeln und die Rentenhöhe festlegen, während die Höhe des Ruhegehaltes von der zuletzt erzielten Besoldung und den ruhegehaltsfähigen Dienstzeiten bestimmt wird.

Im Hinblick auf die hier vorgestellten Befunde ist darauf hinzuweisen, dass die Analysen die statistische Beziehung der Variablen „Lebensarbeitseinkommen“ in der dargestellten Form und „Lebenserwartung" dokumentieren. Die fernere Lebenserwartung der Männer im Alter von 65 Jahren wurde für Rentner und Pensionäre nach dem Verfahren von Chiang (1984) berechnet, in dem für jede Alters- und Lebensarbeitseinkommensklasse (Entgeltpunktquintile und Laufbahngruppen) die Mortalitätsrate als Quotient der Gesamtzahl der Gestorbenen zu der Gesamtzahl der während der Periode gelebten Jahre berechnet wird. Eine solche statistische Beziehung lässt keinen unmittelbaren Rückschluss darauf zu, ob ein höheres Einkommen eine höhere Lebenserwartung erklärt. Dafür gibt es zwei wesentliche Gründe: Zum einen sind Faktoren zu nennen, die sowohl das Einkommen als auch die Sterblichkeit beeinflussen. Ein Beispiel hierfür ist Bildung, für die in internationalen Studien ein kausaler Effekt auf die Sterblichkeit nachgewiesen wurde (Lleras-Muney 2005). Ein höheres Bildungsniveau geht mit einem höheren Lebensarbeitseinkommen und einer höheren Altersrente einher (Fachinger/Himmelreicher 2007; Himmelreicher et al. 2008) und verlängert in der Regel die Lebenserwartung (Shkolnikov et

\footnotetext{
5 Die Anrechnung von Kindererziehungszeiten in den Konten männlicher GRV-Versicherter spielt nahezu keine Rolle. So hatten lediglich 0,2 \% der Männer im Versichertenrentenzugang 2003 eine rentenversicherungsrechtliche Anerkennung von Kindererziehungs- und Berücksichtigungszeiten (Himmelreicher 2006, S. 50).

6 Die Sonderauswertung auf Basis der Statistiken „Personal des öffentlichen Dienstes" und „Versorgungsempfänger des öffentlichen Dienstes", die jährlich vom Statistischen Bundesamt veröffentlicht werden (https://www-ec.destatis.de/ csp/shop/sfg/n0000.csp?treeid=74000), wurde im Rahmen der vorliegenden Untersuchung beim Statistischen Bundesamt in Auftrag gegeben.
} 


\begin{tabular}{|c|c|c|c|c|c|c|c|c|}
\hline \multirow[t]{3}{*}{ Alter } & \multicolumn{2}{|c|}{$\begin{array}{l}\text { Rentner } \\
2002\end{array}$} & \multicolumn{2}{|c|}{$\begin{array}{c}\text { Sterbefälle } \\
\text { Rentner } 2003\end{array}$} & \multicolumn{2}{|c|}{ Pensionäre 2002} & \multicolumn{2}{|c|}{$\begin{array}{c}\text { Sterbefälle } \\
\text { Pensionäre } 2003\end{array}$} \\
\hline & Gesamt & in $\%$ & Gesamt & in $\%$ & Gesamt & in $\%$ & Gesamt & in \% \\
\hline & 5.204 .700 & 100,00 & 256.013 & 100,00 & 481.683 & 100,00 & 22.862 & 100,00 \\
\hline 65-74 Jahre & 3.356 .388 & 64,49 & 90.790 & 35,46 & 277.415 & 57,59 & 5.364 & 23,46 \\
\hline 75-84 Jahre & 1.543 .079 & 29,65 & 106.362 & 41,55 & 157.664 & 32,73 & 9.271 & 40,55 \\
\hline $\begin{array}{l}85 \text { Jahre und } \\
\text { mehr }\end{array}$ & 305.233 & 5,86 & 58.861 & 22,99 & 46.604 & 9,68 & 8.227 & 35,99 \\
\hline
\end{tabular}

\section{Abb. 1: Fernere Lebenserwartung e(65) für Rentner nach Quintilen und Pensionäre nach Laufbahn (2003)}

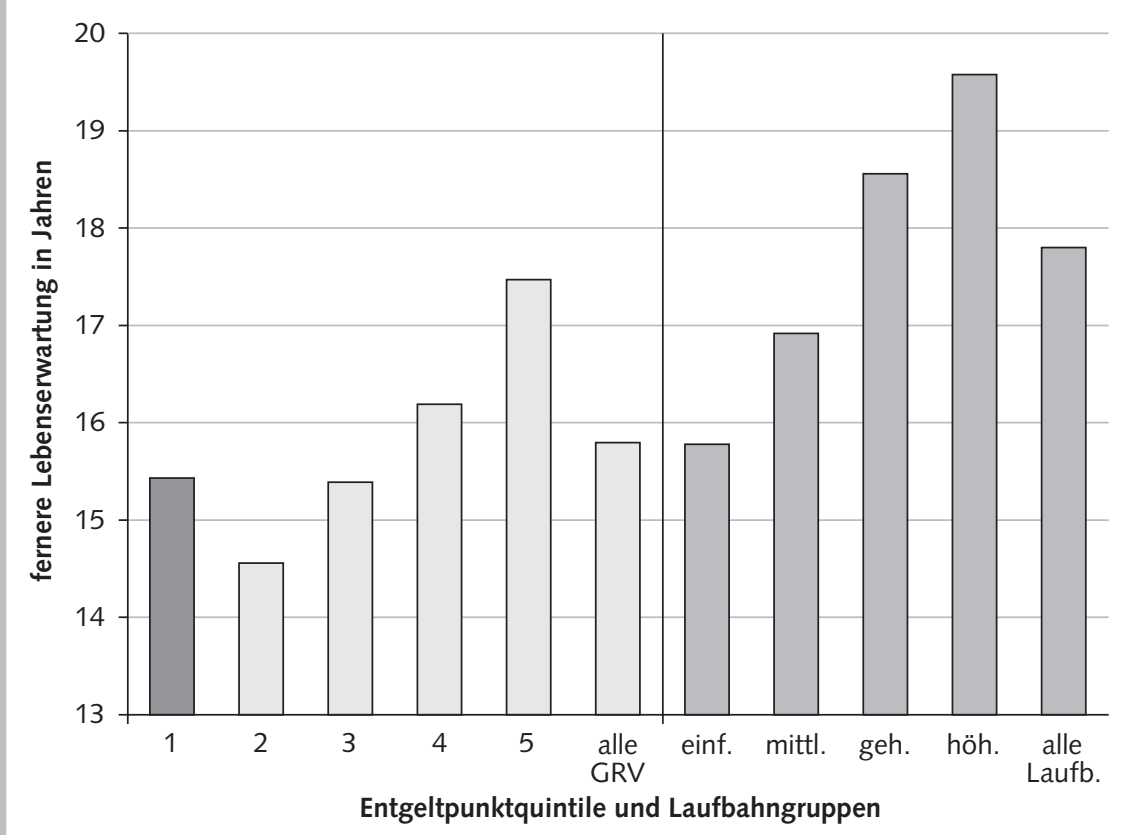

Quelle: FDZ-RV 2003, Fernrechenprojekt u. Sonderauswertung des Statistischen Bundesamtes zu Empfängern von Ruhegehalt nach beamtenrechlichen Vorschriften; Berechnungen und Darstellung der Autoren.

WSI MITTEILUNGEN al. 2007). Allein aufgrund dieser Effekte kann in Studien zu Einkommen und Sterblichkeit ein positiver Zusammenhang der beiden Größen auftreten. Der zweite Grund liegt darin, dass Menschen, die von Geburt an oder während ihrer Erwerbsphase gesundheitliche Beeinträchtigungen ${ }^{7}$ erfahren haben, tendenziell ein geringeres Einkommen erzielen als solche ohne gesundheitliche Probleme. Aufgrund ihres schlechteren Gesundheitszustands weisen sie eine geringere durchschnittliche Lebenserwartung auf. Wiederum muss dies nichts mit dem Einkommen zu tun haben. Anders ausgedrückt lässt sich die Variable „Lebensarbeitseinkommen" als ein aus verschiedenen Variablen bestehendes Konglomerat fassen, das sich unter anderem aus schulischer und beruflicher Bildung, der Lebensführung, dem Gesundheitszustand und aus dem Einkommen zusammensetzt. se 3), denn der Anteil hochaltriger Pensionäre ist höher als jener der Rentner.

Die Analysen mit den Daten des FDZRV ergeben eine durchschnittliche fernere Lebenserwartung der GRV-Rentner von 15,8 Jahren. Die Ergebnisse der Berechnungen finden sich im linken Teil in Abbildung 1 - hier sind auf der Abszisse die fernere Lebenserwartung in Jahren und auf der Ordinate die Quintile der persönlichen Entgeltpunkte abgetragen. Werden die Lebensarbeitseinkommen in Form von Entgeltpunktquintilen in die Berechnungen mit einbezogen, zeigt sich eine positive, statistisch bedeutsame Beziehung zwischen Lebensarbeitseinkommen und Lebenserwartung. ${ }^{8}$ Die Streuung der Lebenserwartung liegt - je nach Entgeltpunktanteil zwischen 14,6 und 17,5 Jahren und beträgt somit 2,9 Jahre. Die Ergebnisse für das zweite bis fünfte Entgeltpunktquintil entsprechen der generellen Annahme einer mit steigendem Lebensarbeitseinkommen steigenden Lebenserwartung. Auf den ersten Blick erstaunlich ist die zu beobachtende höhere durchschnittliche Lebenserwartung in der untersten Einkommensklasse. Dies liegt vermutlich daran, dass ein Teil der dort enthaltenen Rentner höhere Einkünfte außerhalb der Rentenversicherung erzielt hat. $\mathrm{Zu}$ denken ist insbesondere an Beamte, Freiberufler und Selbstständige, die im Laufe ihres Erwerbslebens einen Anspruch auf eine GRV-Rente erworben haben (mindestens Erfüllung der allgemeinen Wartezeit von 60 Monaten), jedoch nicht ausschließlich sozialversicherungspflichtig beschäftigt waren. Häufig erzielen diese Personengruppen höhere Einkommen außerhalb der GRV und sind somit im Hinblick auf ihre Lebenslage und Lebensführung eher mit den Rentnern in den höheren Einkommensklassen mit ähnli-

Empirische Analysen zur differenziellen Sterblichkeit benötigen hohe Fallzahlen, damit hinreichende Zellenbesetzungen, insbesondere bei relativ früh bzw. spät gestorbenen Personen mit spezifischen sozioökonomischen Merkmalen, vorhanden sind. Gerade dann, wenn sozioökonomische Unterschiede der Sterblichkeit untersucht werden, steigen die Anforderungen an die zugrunde liegende Datenbasis erheblich. Tabelle 1 gibt einen Überblick über zentrale Fallzahlen der lebenden und im Folgejahr gestorbenen Rentner und Pensionäre in den beiden Datenbasen. Die Altersdifferenzierungen belegen die höhere Lebenserwartung der Pensionäre (The-

\footnotetext{
7 In beiden Datensätzen sind auch ehemalige Erwerbsminderungsrentner bzw. wegen Dienstunfähigkeit vorzeitig pensionierte Beamte enthalten. Es ist davon auszugehen, dass hierdurch die fernere Lebenserwartung bei Rentnern und Pensionären ab 65 Jahre unterschätzt wird. Hinsichtlich der das 65. Lebensjahr überlebenden Erwerbsminderungsrentner haben diese im Vergleich zu den Altersrentnern eine um 3-4 Jahre geringere fernere Lebenserwartung (vgl. Hoffmann et al. 2006).

8 Zur Signifikanz der Befunde im Hinblick auf die fernere Lebenserwartung der Männer im Alter 65 nach Anzahl der persönlichen Entgeltpunkte vgl. Himmelreicher/Gaudecker 2008, S. $7 f$. Die ermittelten Sterblichkeitsunterschiede sind mit 99\%iger Sicherheit statistisch signifikant.
} 
cher Lebenserwartung vergleichbar. Daraus ergibt sich, dass im ersten Entgeltpunktquintil Personen enthalten sein dürften, deren Lebensarbeitseinkommen untererfasst sind. Die Lebenserwartung dieses Quintils wird deshalb im Folgenden nicht interpretiert. Dieser Befund weist jedoch darauf hin, dass in der GRV wegen ihrer universellen Ausrichtung und vergleichsweise niedriger Barrieren (Wartezeit) unterschiedliche Personenkreise, auch solche mit kürzeren sozialversicherungspflichtigen Erwerbsbiografien und einer nahezu GRV-durchschnittlichen ferneren Lebenserwartung von über 15 Jahren, versichert sind. Insofern bestätigt sich, dass die Heterogenität der Versicherten die Spannweite der Lebenserwartung reduziert (These 1).

Im fünften Quintil, der Gruppe mit der günstigsten sozioökonomischen Lage, also den höchsten persönlichen Entgeltpunkten, wird die höchste Lebenserwartung erreicht. Diese Lebenserwartung liegt ca. zwei Jahre über dem Durchschnitt. Zusammenfassend nimmt die fernere Lebenserwartung vom zweiten zum fünften Entgeltpunktquintil um etwa je ein Jahr zu. Somit leben wohlhabende im Vergleich zu schlechter gestellten Rentnern etwa drei Jahre länger.

In der weiteren Analyse werden den vier Rentnergruppen vier Laufbahngruppen der Pensionäre gegenübergestellt. Die Analysen für Pensionäre ergeben ein ähnliches Bild, jedoch auf höherem Niveau ( $A b$ bildung 1). Ihre fernere Lebenserwartung liegt im Durchschnitt bei 17,8 Jahren. Sie variiert zwischen 15,8 und 19,6 Jahren und steigt mit höherer Laufbahngruppe an, was wir als Einfluss des Lebensarbeitseinkommens auf die Lebenserwartung interpretieren. Die Gruppe mit den höchsten Ruhegehältern, ehemalige Beamte des höheren Dienstes, erreicht die höchste Lebenserwartung. Die Differenz der Lebenserwartungen zwischen den Laufbahngruppen der Pensionäre beträgt 3,8 Jahre. Dies ist deutlich mehr als der bei den Rentnern gemessene Wert von ca. einem Jahr und bestätigt unsere zweite These. Die größere Streuung der Lebenserwartung bei den Pensionären ist ein Indiz für eher homogene Populationen innerhalb der einzelnen Laufbahngruppen.

Insgesamt zeigt sich, dass die Pensionäre im Durchschnitt eine um zwei Jahre höhere Lebenserwartung als die GRVRentner haben (These 3). Bei differenzierter Betrachtung der Lebenserwartung von männlichen Rentnern und Pensionären

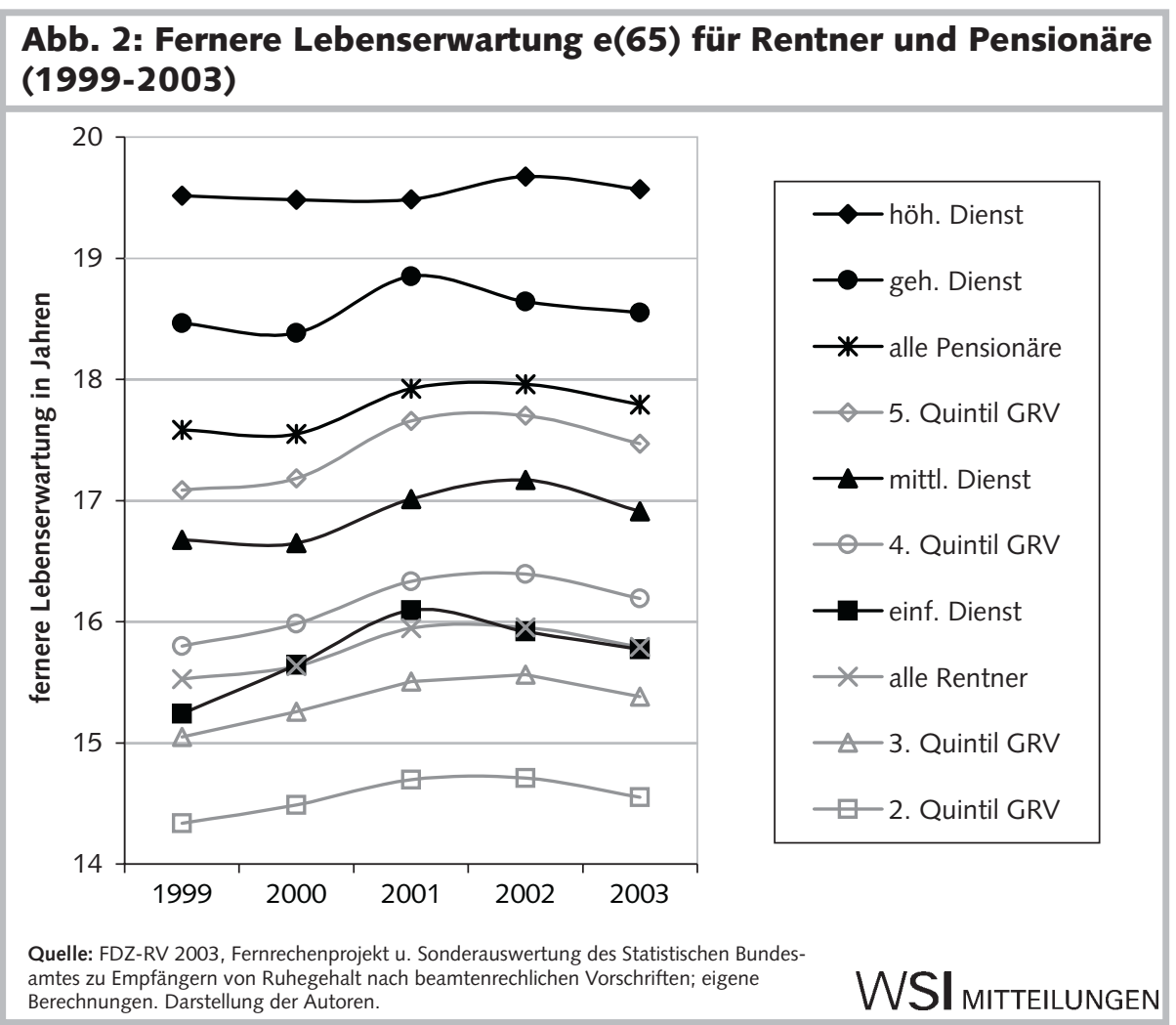

wird deutlich, dass Rentner mit niedrigen Renten und höhere Beamte die Extremwerte der Lebenserwartung mit durchschnittlich etwa 14,6 und 19,6 Jahren bilden. Die durchschnittlich um fünf Jahre höhere Lebenserwartung der höheren Beamten im Vergleich zu den Beziehern niedriger Renten könnte darauf zurückzuführen sein, dass höhere Beamte als Akademiker in Berufen mit geringen physischen und wegen der hohen Arbeitsplatzsicherheit geringeren psychischen Belastungen tätig waren. Im Unterschied dazu handelt es sich bei Niedrigrentnern eher um gering qualifizierte Personen, die ihr Arbeitsleben meist in körperlich belastenderen und vor allem gegen Ende des Erwerbslebens in "unsicheren" Berufen verbracht haben (Himmelreicher et al. 2008). Sie sind überproportional häufig von Arbeitslosigkeit und Abschlägen beim Rentenzugang betroffen und verfügen im Vergleich zu hoch Qualifizierten über wesentlich geringere Lebensarbeitseinkommen (Fachinger/ Himmelreicher 2007).

Die höhere Lebenserwartung von höheren Beamten ist allerdings nicht dieser Gruppe vorbehalten: Auch unter den Rentnern befinden sich viele Personen, die näher an die hohe Lebenserwartung der Pensionäre heranreichen. Der höchste Wert von 19,6 Jahren fernere Lebenserwartung bei Pensionären wird von 112.344 Personen erreicht. Wird bei den Rentnern das 5 . Quintil weiter differenziert, z. B. durch Bildung des 10. Dezils, entfallen auf diese Gruppe fast 600.000 Personen, die dann eine fernere Lebenserwartung von 18,2 Jahren haben.

Abschließend erfolgt ein Vergleich der ferneren Lebenserwartung von Rentnern und Pensionären im zeitlichen Verlauf. Hervorzuheben ist, dass alle untersuchten Gruppen im Fünf-Jahres-Zeitraum eine tendenziell ansteigende Lebenserwartung aufweisen. Die Zunahme der Lebenserwartung von 1999 auf 2003 beträgt bei den Pensionären etwa 2,5 Monate und bei den Rentnern rund drei Monate. Ferner bleiben die Unterschiede zwischen verschiedenen Einkommensgruppen und Laufbahnen weitgehend erhalten. Im Zeitverlauf zeigt sich deutlich, dass die fernere Lebenserwartung aller Rentner sich etwa auf dem Niveau der Pensionäre des einfachen Dienstes bewegt. Allerdings bleibt im Fünf-Jahres-Zeitraum die Lebenserwartung von Männern mit niedrigen Renten niedrig.

Im Hinblick auf die hier dargestellten Befunde ist darauf hinzuweisen, dass andere Analysen zur differenziellen Sterblichkeit trotz unterschiedlicher Datengrundlagen und Forschungsansätze ver- 
gleichbare Ergebnisse erbracht haben (vgl. Helmert 2003; Lampert et al. 2007; ReilHeld 2000).

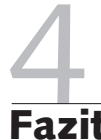

Die auf prozessproduzierten Vollerhebungen basierenden Befunde zur ferneren Lebenserwartung von in Deutschland lebenden männlichen GRV-Rentern und Pensionären verweisen insgesamt darauf, dass ein höheres Lebensarbeitseinkommen, gemessen in Entgeltpunktquintilen und Laufbahngruppen, mit einer Erhöhung der ferneren Lebenserwartung einhergeht. Insofern belegt auch diese Studie den positiven Effekt des Lebensarbeitseinkommens auf die fernere Lebenserwartung von Männern, wobei das Lebensarbeitseinkommen selbst sich aus Merkmalen wie z. B. Periodeneinkommen, Gesundheit, Bildung und Lebensführung zusammensetzt. Die Ergebnisse entsprechen weitgehend denen, die für andere europäische Länder, auch für skandinavische, berichtet werden.

Pensionäre leben im Durchschnitt zwei Jahre länger als Rentner, wobei wiederum während ihres Erwerbslebens besser Gestellte in beiden Gruppen länger leben, d. h., der Einkommenseffekt in Bezug auf die Lebenserwartung gilt bei Rentnern wie Pensionären. Wir gehen davon aus, dass sich die höhere Lebenserwartung der Pensionäre begründen lässt mit den Merkmalen, die eng mit dem Lebensarbeitseinkommen zusammenhängen: Der zwingend erforderliche Gesundheitstest bewirkt eine Selektion der gesünderen Personen bereits zu Beginn der Laufbahn - die unter Beamten wie Pensionären weit verbreitete private Kranken- versicherung verbessert die Versorgung im Krankheitsfall. Hohe Arbeitsplatz- und Einkommenssicherheit ermöglichen einen planbaren Lebensverlauf, was sich wiederum positiv auf die Lebensführung und -zufriedenheit und damit auf die Lebenserwartung auswirkt. Personen, deren Lebensstandard dauerhaft gesichert ist, setzen sich eher mit einer gesunden Lebensführung auseinander und nutzen (Weiter-)Bildungsangebote stärker als Personen, deren vordringlichstes Problem z. B. drohende Arbeitslosigkeit oder die Zahlung der nächsten Miete ist. Hinsichtlich der höheren Lebenserwartung der GRV-Rentner und Pensionäre mit höheren Lebensarbeitseinkommen gehen wir davon aus, dass sie während ihres Erwerbslebens weniger existenziellen Risiken ausgesetzt waren. Zudem verfügen sie meist über Beziehungsnetzwerke, die ihnen eher helfen, Risiken zu bewältigen, aber auch über entsprechende Ressourcen zur Finanzierung zusätzlicher Gesundheitsund Altersvorsorgeprodukte.

Die Lebenserwartung unterscheidet sich zwischen Einkommensgruppen der Rentner in der GRV um rund drei Jahre. Zwischen den Laufbahngruppen der Pensionäre betragen die Unterschiede nahezu vier Jahre. Die größere Streuung der Lebenserwartung bei Pensionären im Unterschied zu GRV-Rentnern führen wir auf eine homogenere Zusammensetzung der Population in den einzelnen Laufbahngruppen zurück - in Kontrast zu den heterogen besetzten Entgeltpunktquintilen der Rentner. Während die Männer in den verschiedenen Laufbahngruppen jeweils über einen ähnlichen Bildungsgrad und einen vergleichbaren sozialen Status verfügen, können GRV-Rentner mit vergleichbaren Entgeltpunkten verschiedene Lebens- und Arbeitsweisen praktiziert haben.
Im Verlauf von fünf Jahren zeigt sich eine um annähernd drei Monate ansteigende Lebenserwartung für beide Gruppen, wobei die Unterschiede zwischen den Einkommensgruppen und Laufbahnen weitgehend erhalten bleiben. Dies zeigt, dass zwar die Lebenserwartung insgesamt steigt, eine Annäherung zwischen den Gruppen jedoch nicht erreicht wird, sodass von einer Reduzierung der sozialen Ungleichheit der Lebenserwartung nicht gesprochen werden kann.

Vor dem Hintergrund des demografischen Wandels, der Lage auf dem Arbeitsmarkt, der Folgen der jüngsten Reformen im deutschen Alterssicherungssystem sowie der Veränderungen im Gesundheitswesen ist anzunehmen, dass die Ungleichheit der Alterseinkünfte und der gesundheitlichen Versorgung zukünftig zunimmt. Infolgedessen könnte es zu einer weiteren Ausweitung der sozialen Ungleichheit der Lebenserwartung kommen. Zahlreiche weitere Faktoren, wie z. B. Bildungs- und Ausbildungschancen, Berufseintritt, Beschäftigung im Lebensverlauf und im Alter, beeinflussen das Lebensarbeitseinkommen und, wie gezeigt werden konnte, damit auch die Lebenserwartung. Dennoch gibt es in Deutschland lediglich Einzelmaßnahmen zur Verringerung der gesundheitlichen Ungleichheit, konkrete Zielvorgaben oder umfassende politische Strategien existieren hierzulande nicht. Angesichts zunehmender Forschungstätigkeit im Bereich der Mortalität ist zu hoffen, dass ein wachsendes öffentliches Problembewusstsein und politische Anstrengungen zur Reduzierung der Sterblichkeitsdifferenzen entstehen. Hierbei können Erfahrungen aus anderen Ländern genutzt werden (Mielck 2006).

\section{LITERATUR}

\begin{abstract}
Alterssicherung in Deutschland (ASID '03) (2005): Zusammenfassung wichtiger Untersuchungsergebnisse, Bundesministerium für Gesundheit und Soziale Sicherung, http://www.bmas.de/coremedia/generator/ 2786/property=pdf/alterssicherung_berichtsband_z_zusammenfassung der_wichtigsten_ergebnisse.pdf
\end{abstract}

Bäcker, G./Bispinck, R./Hofemann, K./Naegele, G. (2000): Sozialpolitik und soziale Lage, Bd.2, Gesundheit und Gesundheitssystem, Familie, Alter, Soziale Dienste, Wiesbaden
Bieber, U./Klebula, D. (2005): Erste Ergebnisse aus der Studie Alterssicherung in Deutschland 2003. Ergebnisse zur Vielschichtigkeit der monetären Situation im Alter, in: Deutsche Rentenversicherung 6-7, S. 362-374 Chiang, L. C. (1984): The Life Table and its Applications, Malabar Deutsche Rentenversicherung Bund (2005): Codeplan Demographiedatensatz: Rentenwegfall und Rentenbestand 1993-2003, FDZ-RV SUFRTBNXXVWITD und FDZ-RV - SUFRTWFXXVWITD 
Doblhammer, G./Rau, R./Kytir J. (2005): Trends in educational and occupational differentials in all-cause mortality in Austria between 1981/82 and 1991/92, Wiener Klinische Wochenschrift 13, S. 468-479 Fachinger, U./Himmelreicher, R. K. (2007): Alterslohnprofile und Qualifikation in den alten Bundesländern - Eine Analyse auf Datenbasis des Längsschnittdatensatzes SUFVVL2004, in: Deutsche Rentenversicherung $11-12$, S. $750-770$

Gaudecker, H.-M. von/Scholz, R. D. (2006): Lifetime Earnings and Life Expectancy, MEA-Discussion Paper 101-06, http://www.mea.unimannheim.de/publications/meadp_102-06.pdf

Gaudecker, H.-M. von/Scholz, R. D. (2007): Differential mortality by lifetime earnings in Germany, in: Demographic Research 4, S. 83-108 Hagen, C./Himmelreicher, R. K./Hoffmann, H. (2007): Typologie des (Mehrfach-) Rentenbezugs, in: RVaktuell 3, S. 52-60

Haider, S./Solon, G. (2006): Life-Cycle Variation in the Association between Current and Lifetime Earnings, in: American Economic Review 4, S. $1308-1320$

Helmert, U. (2003): Subjektive Einschätzung der Gesundheit und Mortalitätsentwicklung, in: Gesundheitswesen 1, S. 47-54

Himmelreicher, R. K. (2006): Analysepotential des Scientific Use Files Versichertenrentenzugang, in: Forschungsrelevante Daten der Rentenversicherung. Bericht vom zweiten Workshop des Forschungsdatenzentrums der Rentenversicherung (FDZ-RV) vom 27. bis 29. Juni 2005 in Würzburg, DRV-Schriften Band 55/2005, Berlin, S. 38-92

Himmelreicher, R. K./Frommert, D. (2006): Gibt es Hinweise auf zunehmende Ungleichheit der Alterseinkünfte und zunehmende Altersarmut? Der Einfluss von Erwerbs- und Familienbiografien auf die Rentenhöhe in Deutschland, in: DIW Vierteljahreshefte zur Wirtschaftsforschung 1,

Berlin, S. 108-130

Himmelreicher, R. K./Gaudecker, H.-M. von/Scholz, R. D. (2006): Nutzungsmöglichkeiten von Daten der Deutschen Rentenversicherung über das Forschungsdatenzentrum der Rentenversicherung (FDZ-RV), in: MPIDR Working Paper WP 2006-018, July

Himmelreicher, R. K./Gaudecker, H.-M. von (2008): „Differentielle Sterblichkeit" von Männern: Ein Beispiel der Nutzungsmöglichkeiten des Forschungsdatenzentrums der Rentenversicherung (FDZ-RV), in: Verhandlungsband des 33. DGS-Kongresses "Die Natur der Gesellschaft" in Kassel 2006 (CD), im Erscheinen

Himmelreicher, R. K./Hagen, C./Clemens, W. (2008): Hat das Ausbildungsniveau einen Einfluss auf das individuelle Rentenzugangsverhalten? Working Paper 37, Rat für Sozial- und WirtschaftsDaten (RatSWD), Berlin Hoffmann, H./Kaldybajewa, K./Kruse, E. (2006): Arbeiter und Angestellte im Spiegel der Statistik der gesetzlichen Rentenversicherung: Rückblick und Bestandsaufnahme, in: Deutsche Rentenversicherung 1, S. 24-53

Lampert, T./Kroll, L. E./Dunkelberg, A. (2007): Soziale Ungleichheit der Lebenserwartung in Deutschland, in: Aus Politik und Zeitgeschichte 42, S. $11-18$

Lauterbach, K. (2007): Der Zweiklassenstaat, Berlin Lauterbach, K./Lüngen, M./Stollenwerk, B./Gerber, A./Klever-Deichert, G. (2006): Zum Zusammenhang zwischen Einkommen und Lebenserwartung, in: Studien zu Gesundheit, Medizin und Gesellschaft 1, http://www.uk-koeln.de/kai/igmg/sgmg/2006-01_einkommen_und_ rentenbezugsdauer. pdf
Lleras-Muney, A. (2005): The Relationship between Eduction and Adult Mortality in the United States, in: Review of Economic Studies 1, S. 189221

Luy, M. (2006): Differentielle Sterblichkeit: die ungleiche Verteilung der Lebenserwartung in Deutschland, Rostocker Zentrum - Diskussionspapier 6, http://www.rostockerzentrum.de/publikationen/rz_ diskussionpapier_6.pdf

Mackenbach, J. P./Bos, V./Anderson, O./Cardano, M./Costa, G./ Harding, S./Reid, A./Hemström, Ö./Valkonen, T./Kunst, A. E. (2003): Widening socioeconomic inequalities in mortality in six western European countries, in: International Journal of Epidemiology 5, S. 830-837 Mielck, A (2006): Quantitative Zielvorgaben zur Verringerung der gesundheitlichen Ungleichheit: Lernen von anderen westeuropäischen Staaten, in: Richter, M./Hurrelmann, K. (Hrsg.): Gesundheitliche Ungleichheit - Grundlagen, Probleme, Perspektiven, Wiesbaden, S. 439-451

Reil-Held, A. (2000): Einkommen und Sterblichkeit in Deutschland: Leben Reiche länger?, Institut für Volkswirtschaft und Statistik, Universität Mannheim, Beiträge zur angewandten Wirtschaftsforschung 580-00 Salzmann, T./Kohls, M. (2007): Mortalitätsanalyse mit den Daten der Deutschen Rentenversicherung - Methodische Überlegungen zum Stichprobencharakter, in: Erfahrungen und Perspektiven. Bericht vom dritten Workshop des Forschungsdatenzentrums der Rentenversicherung (FDZRV) vom 26. bis 28. Juni 2006 in Bensheim, DRV-Schriften Band 5/2006, Berlin, S. 185-199

Scholz, R. D. (2006a): Differentielle Mortalität in Deutschland, in: Schmollers Jahrbuch, S. 375-386, Berlin

Scholz, R. D. (2006b): Differentielle Sterblichkeitsanalyse mit den Daten der Deutschen Rentenversicherung, in: Forschungsrelevante Daten der Rentenversicherung. Bericht vom zweiten Workshop des Forschungsdatenzentrums der Rentenversicherung (FDZ-RV) vom 27. bis 29. Juni 2005 in Würzburg, DRV-Schriften Band 5/2005, Berlin, S. 253-266

Scholz, R. D./Shkolnikov, V./Jdanov, D. (2006): Steigt die Lebenserwartung mit der Rentenhöhe? in: Demografische Forschung - Aus Erster Hand 4, S. 1-2

Shkolnikov, V./Scholz, R. D./Jdanov, D./Stegmann, M./Gaudecker, H.-M. von (2007): Length of life and the pensions of five million retired German men, in: European Journal of Public Health, November, S. 1-6 Statistisches Bundesamt (2004): Finanzen und Steuern - Versorgungsempfänger des öffentlichen Dienstes, 2003, Wiesbaden, https://www-ec. destatis.de/csp/shop/sfg/n0000.csp?treeid=74000

Unger, R. (2003): Soziale Differenzierung der aktiven Lebenserwartung im internationalen Vergleich, Wiesbaden

Wolfson, M./Rowe, G./Gentelmen J. F./Tomiak M. (1993): Career Earnings and Death: A Longitudinal Analysis of Older Canadian Men, in: Journal of Gerontology: Social Sciences 4, S. S167-S179

Wübbeke, C. (2006): Der Einfluss betrieblicher Rahmenbedingungen auf Zeitpunkt und Form des Ausscheidens älterer Arbeitnehmerinnen und Arbeitnehmer aus dem Erwerbsleben - Eine Analyse für Westdeutschland auf Basis der IAB-Beschäftigtenstichprobe 1975-1995 mit Ergänzungsteil I, in: Forschungsrelevante Daten der Rentenversicherung. Bericht vom zweiten Workshop des Forschungsdatenzentrums der Rentenversicherung (FDZ-RV) vom 27. bis 29. Juni 2005 in Würzburg, DRV-Schriften Band 5/2005, Berlin, S. 157-174 